not for the whole. There remains enough over to suggest a genuine law of diminishing returns for mental as for material processes.

\section{COROLLARY OF INDEPENDENCE.}

The next and final point to be raised here is a corollary of what has been said. Since a great many abilities depend almost entirely upon the efficiency of the engines involved and this efficiency varies independently from individual to individual, we may conclude that these abilities themselves vary almost independently from individual to individual.

This theorem has, indeed, been called into question. Some authorities have asserted the existence of a general "sensory level" of ability, so that the persons who are successful at one kind of sensory performance will tend to be so at others also. Other writers have adopted a similar position as regards what they call "practical" ability; persons are taken to be either endowed or not endowed with this all round. But no such position would appear to be supported by the available definite evidence. Dr. McCrae, for example, has recently examined the correlations between different tests that have been entitled those of "performance." These, even among persons of comparatively low status, proved to be, in fact, almost independent of each other. Still more striking has been the result of a very valuable investigation by $\mathrm{Mr}$. Philpott. He undertook to test the discrimination of length, a power which obviously possesses great importance in many spheres of industry. He wisely tested this discrimination in two different ways. First, he showed pairs of lines and made the subjects judge which was the longer; and then he gave them single lines and made them, in each case, draw another line as nearly as possible of the same length. As result, these two performances, that seemingly are but manifestations of one and the same power, turned out to be almost entirely independent. Those who were best at judging between the two lines already drawn did not, to any appreciable extent, excel at making a second line equal to a given one. Quite similar results were obtained for the discrimination of angles, as also for perceiving whether a line is straight or not.
Accepting, then, the conclusion that an immense number of abilities vary from one individual to another almost independently of each other, what is the practical result? Let us try to get a notion how such abilities of any person must be distributed in respect of excellence. By all experience, and also by statistical theory into which we cannot enter here, the great bulk of his abilities will tend to be mediocre; that is to say, they will be near the general average of his class. A fair number will be distinctly above this average, and a fair number below. A small number will be much above; and so also below. The whole frequency distribution will, in fact, have a bell-like shape similar to that which was shown by the curves of the tetrad-differences to be expected from sampling errors. At the extreme ends of the distribution will lie a very small number of performances for which the person is, on one side a genius, and on the other an idiot. Every normal man, woman, and child is, then, a genius at something as well as an idiot at something.

It remains to discover what - at any rate in respect of the genius. This must be a most difficult matter, owing to the very fact that it occurs in only a minute proportion out of all possible abilities. It certainly cannot be detected by any of the testing procedures at present in current usage, but these procedures are capable, I believe, of vast improvement.

The preceding considerations have often appealed to me on looking at a procession of the unemployed, and hearing some one whisper that they are mostly the unemployable. That they are so actually I cannot help concurring. But need they be so necessarily? Remember that every one of these, too, is a genius at something-if we could only discover what. I cherish no illusion, indeed, that among them may be marching some " mute inglorious Milton, some Cromwell guiltless of his country's blood." For these are walks in life that appear to involve a large amount of $g$. But I am quite confident that every one of them could do something that would make him a treasure in some great industrial concern; and I see no reason why some should not have even become famous, in such occupations, for example, as those of dancers, jockeys, or players of popular games.

\title{
Radiometric Measurements of Stellar and Planetary Temperatures.
}

By Dr. W. W. Coвlentz, Bureau of Standards, Washington, D.C.

' $\mathrm{H}$ HE recent measurements of planetary radiation and planetary temperatures, especially of Mars, had their beginning in the first really successful tests at the Lick Observatory, Mt. Hamilton, California, in July I9I4, when thermo-couple measurements were made on Ir 2 celestial objects, including I05 stars, down to magnitude $6 \cdot 7$, and the planets Venus, Mars, Jupiter, and Saturn. (Bureau of Standards Sci. Paper, § 244, I9I4.) The experimental procedure then employed, and the results obtained, have foreshadowed, in an unforeseen manner, practically everything that has been accomplished since then.

During the world strife and confusion that occurred in the meantime, nothing further was accomplished until the fall of $\mathrm{I} 92 \mathrm{I}$, when, at the invitation of the Lowell Observatory, I was given a further opportunity to try out a new method of measuring stellar tempera- tures by means of screens of quartz, water, etc., which separated the incident radiation into components containing radiation of the following wave-lengths: 0.3 to $0.43 \mu ; 0.43$ to $0.6 \mu ; 0.6$ to $\mathrm{I} .4 \mu ; \mathrm{I} .4$ to $4 . \mathrm{I} \mu$; and $4 . \mathrm{I}$ to $\mathrm{I} 2 \mu$. In this manner the distribution of energy in the spectra of 16 stars was determined, thereby obtaining for the first time an insight into the radiation intensities in the complete spectrum of a star.

From a comparison of the observed stellar spectral radiation components with similar data, calculated for a black body at various temperatures, it was found that the stellar temperatures range from $2500^{\circ}$ to $3200^{\circ} \mathrm{K}$ for red stars, up to $14,000^{\circ} \mathrm{K}$ or even higher. for blue stars, which is in good agreement with other methods of estimating stellar temperatures.

Since then Pettit and Nicholson, using similar thermocouples and a transmission screen of water attached to 
the great Ioo-inch reflector of the Mt. Wilson Observatory, have been able to study long period variable stars of visual magnitude down to magnitude $9 \cdot 2$ and temperatures below $2000^{\circ} \mathrm{K}$. In the meantime, Abbot has succeeded in measuring the distribution of energy in the spectrum of a number of the brightest stars, and has obtained temperatures in good agreement with those previously observed by the writer, using a thermocouple and transmission screens. Furthermore, using the writer's radiometric measurements, and assuming that the radiation from these stars is similar to that of a black body, Stetson has computed stellar diameters which are in good agreement with those measured directly with an interferometer.

From the foregoing results it appears that the outlook for the thermocouple as a useful instrument in stellar radiometry is very promising: (I) as a device for estimating stellar diameters; and (2) for determining the temperature of stars that are too small to measure the spectral energy distribution directly, thus supplementing the work of Abbot. The only discrepancy is perhaps in the published measurements of the radiation from Sirius. There seems to be something elusive about this star. Back in I9I4 it was on the list for measurement; but because of daylight and low altitude no attempt was made to measure it. In I92I a series of radiometric measurements gave a watercell transmission of about 70 per cent. (in Bur. Stds. Sci. Paper, $\S 438$, is given the uncorrected value, 65 per cent.). This would indicate that the spectral type of the companion star of Sirius is lower than A-perhaps below type $\mathrm{F}$ or down to type $\mathrm{K}$. Owing to an uncertainty in some recent radiometric measurements on this star, further work must be undertaken to disprove or verify these results.

The results of $192 \mathrm{I}$ were so promising that the Lowell Observatory extended the invitation to continue the investigation in 1922 , especially of planetary temperatures, in preparation for the opposition of Mars in 1924.

In all these measurements of planetary radiation, use was made of the 40-inch Lowell reflector with focal lengths of 220 inches and 640 inches; Mr. C. O. Lampland, astronomer at the Lowell Observatory, collaborating. The measurements of 1922 and of 1924 are in agreement in showing that the planetary radiation emanating from Jupiter and from Saturn, and transmitted by our atmosphere, is very small, while the planetary radiation from Venus, Mars, and the moon is, relatively, very intense. The radiometric measurements made in 1922 indicated that about 30 per cent. of the total radiation emanating from Mars is of planetary origin, as compared with 80 per cent. from the moon. From the fact that the surface temperature of the moon is estimated to be considerably above $100^{\circ} \mathrm{C}$., the writer estimated(Bur.Stds. Sci. Paper, $\S 460$, 1922) that the temperature rise of the surface of Mars is considerable-perhaps so high as $10^{\circ}$ to $20^{\circ} \mathrm{C}$.

From the water-cell transmissions obtained by us in 1922, Menzel (Astrophys. Jour., 58, p. 65, 1923), using Russell's formula, calculated the following temperatures: Venus, $50^{\circ} \mathrm{C}$.; Jupiter and Saturn, $-\mathrm{I} 10^{\circ} \mathrm{C}$.; and the moon, $120^{\circ} \mathrm{C}$. From the water-cell transmissions of the radiation from Mars, observed on June $I_{5}$ and $\mathrm{r} 8, \mathbf{1 9 2 2}$, his calculations gave temperatures of $-5^{\circ} \mathrm{C}$. and $-9^{\circ} \mathrm{C}$. respectively, the higher value being for the smaller receiver, which intercepted 0.4 of the diameter of the planetary disk. From the large temperature gradient observed in 1924 , extending from the equator to the poles and the limbs of the planet, it is evident that the highest temperature, $-5^{\circ} \mathrm{C}$., is an average value, which should be corrected for latitude. This would raise Menzel's values to $5^{\circ} \mathrm{C}$., or perhaps even higher, when corrected for non-black body radiation.

Using our data observed in 1924, Menzel has calculated an average temperature of $-30^{\circ} \mathrm{C}$. for the whole disk of Mars. The temperatures of the apparent centre of the disk ranged from $-5^{\circ} \mathrm{C}$. on the bright areas to $22^{\circ} \mathrm{C}$. on the adjacent dark areas, the predominating values being $6^{\circ}$ to $9^{\circ} \mathrm{C}$. The true temperatures would be about $10^{\circ}$ higher, or $15^{\circ}$ to $20^{\circ} \mathrm{C}$. The temperatures derived by this method of reducing the data are in good agreement with those obtained by three other methods, all of which indicate conclusively that the equatorial temperature of Mars, at perihelion, was considerably above $0^{\circ} \mathrm{C}$.

The planet Venus is one of the most interesting cases met with in radiometric observations. The surface of the planet is hidden by clouds, and its period of rotation is undetermined. Hence the thermocouple radiometer appears to be a means of obtaining further information on this question. For it was found that not only does the illuminated crescent show the presence of considerable planetary radiation, but the unilluminated part of the disk also emits a large amount of infra-red rays. This raises the question whether the radiation from the dark side of the planet is owing to a rapid rotation. If the period of rotation is long (225 days), then it seems necessary to assume that the surface of Venus is still quite warm; although the highly selective condition of the planetary radiation at 8-I $2 \mu$ may perhaps be interpreted as due to radiation of the hot gases convected from the illuminated over the dark part of the planetary surface. However, since this involves distances of 800 to rooo miles over the surface, it seems difficult to reconcile this assumption with the radiative properties of the gases. which probably constitute the atmosphere of Venus.

An interesting and important observation is that the intensity of the radiation emitted from near the south cusp in the present position of the planet Venus-both for the dark and the illuminated regions - is greater than that emanating from corresponding points near the north cusp. This may be owing to differences in the surface conditions, as previously observed on Mars. Then, again, it suggests an effect of insolation due to inclination of the axis of rotation, analogous to seasonal changes on Mars and the earth. Further radiometric observations, at different presentations of the planet's surface to the earth, will be required to test this question. If this is seasonal, then it should be possible.to establish the position of the axes of rotation of Venus.

From our data observed in 1924, Menzel calculated the following temperatures: Venus, $60^{\circ} \mathrm{C}$.; Jupiter, $-135^{\circ}$ C. ; Saturn, $-150^{\circ}$ C. ; Uranus, lower than $-\mathrm{I} 85^{\circ} \mathrm{C}$.; and the moon, $125^{\circ} \mathrm{C}$. These calculations are in good agreement with those based upon our data of I922, and they are in good agreement with Christiansen's theoretical calculations made long ago, in which he obtained the following values: Venus, $65^{\circ} \mathrm{C}$.; Jupiter, $-147^{\circ} \mathrm{C}$.;

$$
\text { No. } 2916 \text {, VOL. I I6] }
$$


Saturn, $-180^{\circ}$ C.: Uranus, $-207^{\circ}$ C. ; and Neptune, $-221^{\circ} \mathrm{C}$.

In the case of Saturn, the observations seem to indicate that the temperature is higher than can be maintained solely by the incident solar radiation. In other words, the interior of the ball of Saturn is still relatively hot. This is in agreement with the views expressed by Poynting twenty years ago, when he made his theoretical calculations of planetary temperatures, obtain- ing $69^{\circ} \mathrm{C}$. for Venus and $-38^{\circ} \mathrm{C}$. for Mars. As an average value for the whole disk of Mars, Poynting's calculation of $-38^{\circ} \mathrm{C}$. is in good agreement with our observed value of $-30^{\circ} \mathrm{C}$.

The observed temperature of the moon, $125^{\circ} \mathrm{C}$., is in good agreement with the values assigned to it years ago by Lord Rosse, $110^{\circ} \mathrm{C}$., and more recently by Very, I $50^{\circ} \mathrm{C}$., by comparison of the lunar radiation against a black body.

\section{Deinosaur Eggs.}

By Dr. F. A. Bather, F.R.S.

THE Times published on September 9 an interesting despatch from its Peking correspondent, giving further details of the discoveries in Mongolia by the expedition from the American Museum of Natural History, led by Mr. Roy C. Andrews. The fresh information about the fossil eggs attributed to deinosaurs suggests some comment.

Two of the recent finds are particularly worthy of note. One is a nest of twelve eggs almost perfectly preserved. The eggs were arranged in a circle, with the narrow ends pointing outwards; no doubt the broad end was, as in birds, that which first emerged, but in birds' nests this end of the egg is, as one would expect, directed outwards. Then the eggs are said to have been " in two layers of six each," those of the upper layer presumably alternating with those of the under one. This, if a correct description, suggests that the deinosaur scooped a basin in the sand, and perhaps covered the first layer with sand, as is the habit of the Nile crocodile.

The other find was a nest of five eggs, "smaller than those found two years ago, more elongated, and evidently the produce of another type of dinosaur. The shells are smooth, in contrast to the dimpled surface of the others, and very thin." Are they the eggs of a deinosaur at all? All as yet known have notably thick shells. These might be the eggs of a crocodile or a chelonian. Turtles' eggs are now being fossilised in just the same way in the sand-dunes of Western Australia.

The last question will have to be settled by the study of thin sections. It is strange how little was known two years ago about the structure of egg-shells, whether recent or fossil, of birds or of reptiles. Nathusius alone has studied thin sections of recent egg-shells, describing that of the moa in 1870 , and that of a python in I883. About I870 also, some Cretaceous fresh-water sandstones in Provence yielded the bones of a reptile and some fragments of egg-shell. The latter and thin sections cut from them were re-studied in 1922 by Dr. Van Straelen and Mr. E. Deneyer of Brussels. The reptile is generally supposed to be some sort of deinosaur, and the eggs are assigned to it with some probability, made stronger by Dr. Van Straelen's later researches, of which a preliminary account was recently issued by the American Museum of Natural History. The following summary is based on the researches quoted and a few more general facts.

The egg of all birds and reptiles, when first formed within the body, is covered with a flexible membrane. At a large number of points in this membrane minute specks of lime appear. Round each of these specks more lime is gradually added in successive layers like the coats of an onion, thus building up little spheroids. When these spheroids meet one another, they form a complete coating to the egg, so that further layers of lime can be laid down only on the outer side of this. The layers outside any one spheroid thus build up a pile of irregularly prismatic shape and connect irregularly with the layers of the adjacent piles or prisms. On the inner surface of the first-formed coat each spheroid appears as a little hillock or mamilla ; so this is called the " mamillar zone." 'The outer layers form the "prismatic zone." Outside the latter some of the membrane may remain as a "cuticle."

In chelonians the egg-shell is very thin, being composed only of the mamillar zone, with gaps remaining between the spheroids. In snakes the shell is still thin; the prisms are there, but they do not always join up. In crocodiles the shell is thicker than in turtles and in most birds, but the prismatic zone is scarcely developed; spaces, in the form of canals of two sizes, are left at intervals between the spheroids, so that air can reach the developing embryo. All birds have a mamillar zone and a relatively thick prismatic zone. In the Ratitæ the shell is thick and the air-canals branch as they near the outside, each ending in a group of pores. In the higher birds the shell is thinner and each canal opens in a single pore.

The deinosaur egg-shells already described by Dr. Van Straelen fall into three groups. In those associated with Protoceratops, from the base of the Upper Cretaceous, very small spheroids form a thin mamillar zone ; the thick prismatic layer is traversed by relatively few air-canals, which end in minute pores and are of the same width throughout. In a Mongolian shell thought to belong to a duck-billed deinosaur the spheroids are larger, the pores a little larger, and the canals swell in the middle. In the shell from Provence the mamillar zone is much as in the last-mentioned; but above it are cavities which give rise to straight air-canals of equal bore, ending in pores of $0.1 \mathrm{~mm}$. to $0.2 \mathrm{~mm}$. diameter.

Thus the three types of supposed deinosaur eggs show a general agreement with one another. They differ from the eggs of all other creatures, but present certain resemblances to the eggs of crocodiles and birds, just as one would expect deinosaur eggs to do. We may therefore conclude that they really were laid by the deinosaurs the remains of which are found with them.

In addition to these three types, Dr. Van Straelen has in hand material from other parts of the world, including a probable deinosaur egg from the Oxford Clay of Peterborough and other eggs from the Oolite

$$
\text { No. } 2916 \text {, vOL. I I 6] }
$$

